\title{
Surface-based shape classification using Wasserstein distance
}

\author{
Ming Ma*, Na LeI ${ }^{\dagger}$, Kehua Su ${ }^{\ddagger}$, Junwei Zhang ${ }^{\S}$, \\ Chengfeng Wen* ${ }^{*}$, Li Cui ${ }^{\uparrow}$, Xin Fan ${ }^{\dagger}$, And Xianfeng Gu*
}

\begin{abstract}
Surface based shape analysis plays a fundamental role in computer vision and medical imaging. In this work, we proposes a novel method for shape classification of brain's hippocampus using Wasserstein distance based on optimal mass transport theory. In comparison with the conventional method based on MongeKantorovich theory, our proposed method employs Monge-Brenier theory for the computation of the optimal mass transport map, which remarkably ameliorates the efficiency by reducing computational complexity from $O\left(n^{2}\right)$ to $O(n)$. Using the conformal mapping, our method maps the metric surface with disk topology to the unit planar disk, which pushes the area element on the surface to the disk and incurs the area distortion. A probability measure is then determined by this area distortion. Given any two probability measures on two surfaces, our method is capable of obtaining a unique optimal mass transport map between them. The transportation cost of this optimal mass transport defines the Wasserstein distance between two surfaces, which intrinsically measures the dissimilarities between surface based shapes and thus can be used for shape classification. Experimental results on surface based hippocampal shape analysis demonstrates the efficiency and efficacy of our proposed method.
\end{abstract}

\section{Introduction}

The past decades have witnessed a remarkable growth of interest and research efforts in surface representations for 3D shape analysis. Some of conventional methods focus on mathematical morphology [20], medical axis [5]

\footnotetext{
*The author is with Department of Computer Science, Stony Brook University.

†The author is with Dalian University of Technology.

$\ddagger$ The author is with Wuhan University.

$\S$ The author is with Stony Brook University.

T The author is with School of Mathematical Sciences, Beijing Normal University.
} 
and volume measurements [21]. More recently, surface based methods, such as conformal mapping based shape analysis using Ricci flow [29], surface matching [30] and anatomical morphometry analysis [15], have also attracted increasing interest and attention. In this paper, we cope with a challenging problem of 3D shape analysis and classification [23, 4, 3]. Some of related research works employ landmark-based descriptor, medial axes, and deformation field. Some of others utilize surface-based shape classification methods and brain morphometry [25, 24, 14]. To address the 3D shape classification problem by following the same spirit of surface based method, we propose a Wasserstein distance method based on Monge-Brenier optimal mass transport (OMT) theory for shape classification of brain's hippocampus in epilepsy. Our method first employs conformal mapping to map the metric surface with disk topology to the unit planar disk, and then acquire the probability measure by the induced area distortion. By computing an unique optimal mass transport map between two surfaces equipped with two probability measures, we can obtain the optimal transportation cost which defines the Wasserstein distance between two surfaces. This Wasserstein distance can intrinsically measure the dissimilarities between surface based shapes, and thus can be employed for shape classification.

Contributions To the best of our knowledge, this work is the first one to propose a novel shape classification method using Wasserstein distance based on Monge-Brenier theory for studying hippocampus of brain. We enumerate contributions of this work as follows:

- proposes a novel shape classification method using Wasserstein distance based on optimal mass transport theory.

- demonstrates the potential of our method on the discriminative analysis of hippocampal shape in epilepsy.

The remainder of this paper is organized as follows. Section 2 reviews related works of $3 \mathrm{D}$ shape analysis and classification. Section 3 briefly introduces the theoretic background of Optimal Mass Transport theory and Wasserstein distance. Section 4 gives the algorithmic implementation details for optimal mass transport map and computation of Wasserstein distance, followed by experimental results in Section 5 .

\section{Previous work}

\subsection{Shape classification}

Shape classification and brain morphometry studies have obtained extensive research interest in shape analysis and medical imaging fields in the 
past decades. A surface-based representation and classification technique was studied for hippocampal shape analysis in [25] based on fine-scale spherical harmonic expansion. A new framework for 3D surface object classification using spherical harmonic parameterization and point distribution model was presented in [26]. In [24], shape were represented by computing the histogram of pairwise diffusion distances between all points. A novel method called compact Shape-DNA was proposed in [14] to describe the shape of a triangular surface mesh for shape comparison and classification.

\subsection{Optimal mass transport}

In the past decades, numerous research works have investigated optimal mass transport problem. A image registration and warping method was proposed using optimal mass transport [19], which is based on a partial differential equation approach to the minimization of the $L^{2}$ Kantorovich-Wasserstein under a mass preservation constraint. A texture mapping method based on optimal mass transport was presented in [13]. This approach first computed an angle-preserving mapping and then corrected it using the mass transport procedure derived via a certain gradient flow. To deal with $2 \mathrm{D}$ shape reconstruction and simplification problem, [11] presented a novel optimal transport driven approach, where the input point was approximated by a simplicial complex considered as a sum of uniform measures. For image processing, [10] devised a novel method by converting capacity-constrained Voronoi tessellation into an optimal transport problem, and exploited the variational nature of this formulation to design an efficient optimization technique of point distributions via constrained minimization in the space of power diagrams. In brain morphological study and visualization, [27] proposed an area-preserving mapping method computed using a optimal mass transport, and applied this method to cortical surface classification for recognition of Alzheimer's Disease.

\section{Theoretic background}

This section briefly introduces the theoretic background of Optimal Mass Transport theory and Wasserstein distance. We refer readers to a classical textbook [18] for conformal geometry, the seminal papers [22] on optimal transport map with Kantorovich's method, and [17] for more detailed proofs of the proposed method.

\subsection{Optimal mass transport}

Monge [6] raised the optimal mass transport problem in the 18th century. 
Definition 3.1 (Optimal Mass Transport). Suppose $(X, \mu),(Y, \nu)$ are metric spaces with probability measures, which have the equal total mass $\int_{X} \mu d x=$ $\int_{Y} \nu d y$. A map $T: X \rightarrow Y$ is measure preserving, if for any Boreal set $B \subset$ $Y, \mu\left(T^{-1}(B)\right)=\nu(B)$. Given a transportation cost function $c: X \times Y \rightarrow \mathbb{R}$, find the measure preserving map $T: X \rightarrow Y$ that minimizes the total transportation cost

$$
\mathcal{C}(T):=\int_{X} c(x, T(x)) d \mu(x) .
$$

The problem of finding a map $T$ minimizing Eqn. 1 (such that $\nu=T_{\sharp} \mu$ ), has been first studied by Monge [6] in the 18th century. At the end of 1980's, Brenier [7] has proved that there is a convex function $f: X \rightarrow R$, and the optimal mass transport map $T(x)$ is given by the gradient map $x \rightarrow \nabla f(x)$.

\subsection{Discrete optimal mass transport}

Suppose $\mu$ has compact support on $X$. Define $\Omega=\operatorname{Supp} \mu=\{x \in X \mid \mu(x)>$ $0\}$, and assume $\Omega$ is a convex domain in $X$. The space $Y$ is discretized to $Y=\left\{y_{1}, y_{2}, \cdots, y_{k}\right\}$ with Dirac measure $\nu=\sum_{j=1}^{k} \nu_{j} \delta\left(y-y_{j}\right)$. We define a height vector $\mathbf{h}=\left(h_{1}, h_{2}, \cdots, h_{k}\right) \in \mathbb{R}^{k}$, consisting of $k$ real numbers. For each $y_{i} \in Y$, we construct a hyperplane defined on $X$,

$$
\pi_{i}(\mathbf{h}):\left\langle x, y_{i}\right\rangle+h_{i}=0 .
$$

We define a piece-wise linear convex function

$$
u_{\mathbf{h}}(x)=\max _{i=1}^{k}\left\{\left\langle x, y_{i}\right\rangle+h_{i}\right\}
$$

We denote its graph by $G(\mathbf{h})$, which is an infinite convex polyhedron with supporting planes $\pi_{i}(\mathbf{h})$. The projection of $G(\mathbf{h})$ induces a polygonal partition of $\Omega$,

$$
\Omega=\bigcup_{i=1}^{k} W_{i}(\mathbf{h}),
$$

where each cell $W_{i}(\mathbf{h})$ is the projection of a facet of the convex polyhedron $G(\mathbf{h})$ onto $\Omega$,

$$
W_{i}(\mathbf{h})=\left\{x \in X \mid u_{\mathbf{h}}(x)=\left\langle x, y_{i}\right\rangle+h_{i}\right\} \cap \Omega .
$$




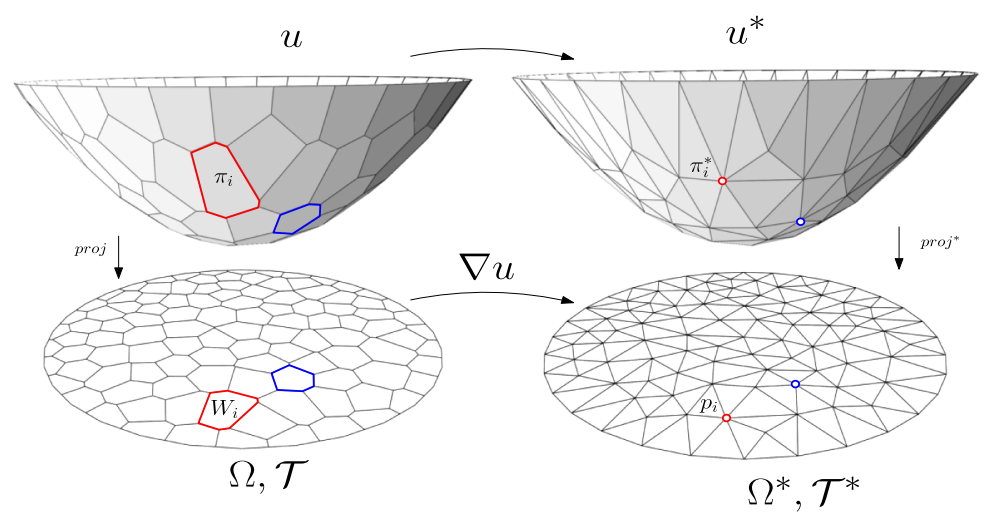

Figure 1: Discrete optimal mass transport map with Brenier's approach.

This partition is equivalent to a power diagram, denoted as $D(\mathbf{h})$, as explained in [17]. The area of $W_{i}(\mathbf{h})$ is given by

$$
w_{i}(\mathbf{h})=\int_{W_{i}(\mathbf{h})} \mu(x) d x .
$$

The convex function $u_{\mathbf{h}}$ on each cell $W_{i}(\mathbf{h})$ is a linear function $\pi_{i}(\mathbf{h})$, therefore, the gradient map

$$
\nabla u_{\mathbf{h}}: W_{i}(\mathbf{h}) \rightarrow y_{i}, i=1,2, \cdots, k
$$

maps each $W_{i}(\mathbf{h})$ to a single point $y_{i}$. The discrete OMT with Brenier's approach is shown in Figure 1.

The following theorem plays a fundamental role for discrete optimal mass transport theory.

Theorem 3.1. Given a convex domain $\Omega \subset \mathbb{R}^{n}$, with measure density $\mu$ : $\Omega \rightarrow \mathbb{R}$, and a discrete point set $Y=\left\{y_{1}, \cdots, y_{k}\right\}$ with discrete measures $\nu=$ $\left\{\nu_{1}, \cdots, \nu_{k}\right\}$. Suppose $\sum_{j=1}^{k} \nu_{j}=\int_{\Omega} \mu, \nu_{j}>0$. Then there must exist a height vector $\mathbf{h}=\left\{h_{1}, \cdots, h_{k}\right\}$ unique up to translations, such that the convex function Eqn. 3 induces the cell decomposition of Eqn. 4. The following area-preserving constraints are satisfied for all cells,

$$
\int_{W_{i}(\mathbf{h})} \mu(x) d x=\nu_{i}, i=1,2, \cdots, k .
$$


Furthermore, the gradient map grad $u_{\mathbf{h}}$ optimizes the following transportation cost

$$
\mathcal{C}(T):=\int_{\Omega}|x-T(x)|^{2} \mu(x) d x
$$

The existence and uniqueness was first proven by Alexandrov [1] using a topological method; the existence was also proven by Aurenhammer, Hoffmann and Aronov [2], the uniqueness and optimality was proven by Brenier [7]. Recently, Gu et al. [17] gives a novel proof for the existence and uniqueness based on the variational principle, which leads to the computational algorithm directly.

Define the admissible space of height vectors:

$$
H_{0}:=\left\{\mathbf{h} \mid \sum_{j=1}^{k} h_{j}=0 \text { and } \int_{W_{i}(\mathbf{h})} \mu>0, \forall i=1, \cdots, k,\right\} .
$$

Then define the energy $E(\mathbf{h})$,

$$
E(\mathbf{h})=\int_{\Omega} u_{\mathbf{h}}(x) \mu(x) d x-\sum_{i=1}^{k} \nu_{i} h_{i}
$$

or equivalently

$$
E(\mathbf{h})=\int_{\mathbf{0}}^{\mathbf{h}} \sum_{i=1}^{k} w_{i}(\eta) d \eta_{i}-\sum_{i=1}^{k} \nu_{i} h_{i}+C
$$

where $C$ is a constant. Consider the shape bounded by the graph $G(\mathbf{h})$, the horizontal plane $\left\{x_{n+1}=0\right\}$ and the cylinder consisting of vertical lines through $\partial \Omega$, the volume of the shape is given by the first term.

The gradient of the energy is given by

$$
\nabla E(\mathbf{h})=\left(w_{1}(\mathbf{h})-\nu_{1}, \cdots, w_{k}(\mathbf{h})-\nu_{k}\right)^{T}
$$

Suppose the cells $W_{i}(\mathbf{h})$ and $W_{j}(\mathbf{h})$ intersects at an edge $e_{i j}=W_{i}(\mathbf{h}) \cap$ $W_{j}(\mathbf{h}) \cap \Omega$, then the Hessian of $E(\mathbf{h})$ is given by

$$
\frac{\partial^{2} E(\mathbf{h})}{\partial h_{i} \partial h_{j}}=\left\{\begin{array}{ll}
\frac{\int_{e_{i j}} \mu(x) d x}{\left|y_{j}-y_{i}\right|} & W_{i}(\mathbf{h}) \cap W_{j}(\mathbf{h}) \cap \Omega \neq \emptyset \\
0 & \text { otherwise }
\end{array} .\right.
$$


The following theorem lays down the theoretic foundation of our OMT map algorithm.

Theorem 3.2 (Discrete Optimal Mass Transport [17]). If $\Omega$ is convex, then the admissible space $H_{0}$ is convex, so is the energy (Eqn. 10). Moreover, the unique global minimum $\mathbf{h}_{0}$ is an interior point of $H_{0}$. And the gradient map (Eqn. 7) induced by the minimum $\mathbf{h}_{0}$ is the unique optimal mass transport map, which minimizes the total transportation cost (Eqn. 9).

The proof of Theorem 3.2 is reported in [17]. Due to the convexity of the volume energy Eqn. 10, the global minimum can be obtained efficiently using Newton's method. Comparing to Kantorovich's approach, where there are $O\left(n^{2}\right)$ unknowns, this approach has only $O(n)$ unknowns.

\subsection{Wasserstein metric space}

Suppose $(M, \mathbf{g})$ is a Riemannian manifold with a Riemannian metric $\mathbf{g}$.

Definition 3.2 (Wasserstein Space). Let $\mathcal{P}_{p}(M)$ denote the space of all probability measures $\mu$ 's on $M$ with finite $p^{\text {th }}$ moment, where $p \geq 1$. Suppose there exists some point $x_{0} \in M$ that $\int_{M} d\left(x, x_{0}\right)^{p} d \mu(x)<+\infty$, where $d$ is the geodesic distance induced by $\mathbf{g}$.

Given two probability $\mu$ and $\nu$ in $\mathcal{P}_{p}$, the Wasserstein distance between them is defined as the transportation cost induced by the optimal mass transport map $T: M \rightarrow M$,

$$
W_{p}(\mu, \nu):=\inf _{T_{\#} \mu=\nu}\left(\int_{M} d(x, T(x))^{p} d \mu(x)\right)^{\frac{1}{p}} .
$$

The following theorem plays a fundamental role for the current work.

Theorem 3.3. The Wasserstein distance $W_{p}$ is a Riemannian metric of the Wasserstein space $\mathcal{P}_{p}(M)$.

Detailed proof can be found in [28].

\section{Algorithms}

This section gives the algorithmic implementation details for optimal mass transport map (OMT-Map) generation using our new variational framework. Based on the OMT-Map algorithm, we introduce the computation for conformal Wasserstein distance between surfaces. 


\subsection{Optimal Mass Transport Map (OMT-Map) Algorithm}

Assume $\Omega$ is a convex planar domain with measure density $\mu . P=\left\{p_{1}, \cdots, p_{k}\right\}$ is a point set with measure $\nu=\left\{\nu_{1}, \cdots, \nu_{k}\right\}$, such that $\int_{\Omega} \mu(x) d x=\sum_{i=1}^{k} \nu_{i}$.

According to the discussion in previous section, the OMT-Map can be obtained by minimizing the convex energy in Eqn. 10. In practice, the energy can be optimized using Newton's method, which requires the computation of the energy gradient using Eqn. 12, and the Hessian matrix using Eqn. 13. The method is straightforward, but the initialization and the step length selection need to be specially addressed.

Initialization By translating and scaling, $P$ could be inside $\Omega, P \subset \Omega$. At the beginning, we set each power weight $h_{i}$ to be 0 , namely $\mathbf{h}=\mathbf{0}$, and compute the power diagram $D(P, \mathbf{h})$ and the Delaunay triangulation $T(P, \mathbf{h})$. In this scenario, $D(P, \mathbf{h})$ is a conventional voronoi diagram.

Step Length Selection Suppose at the $k$-th step in the optimization, the power weight vector is $\mathbf{h}^{k}$, and all Voronoi cells $W_{i}\left(\mathbf{h}^{k}\right)$ are non-empty.

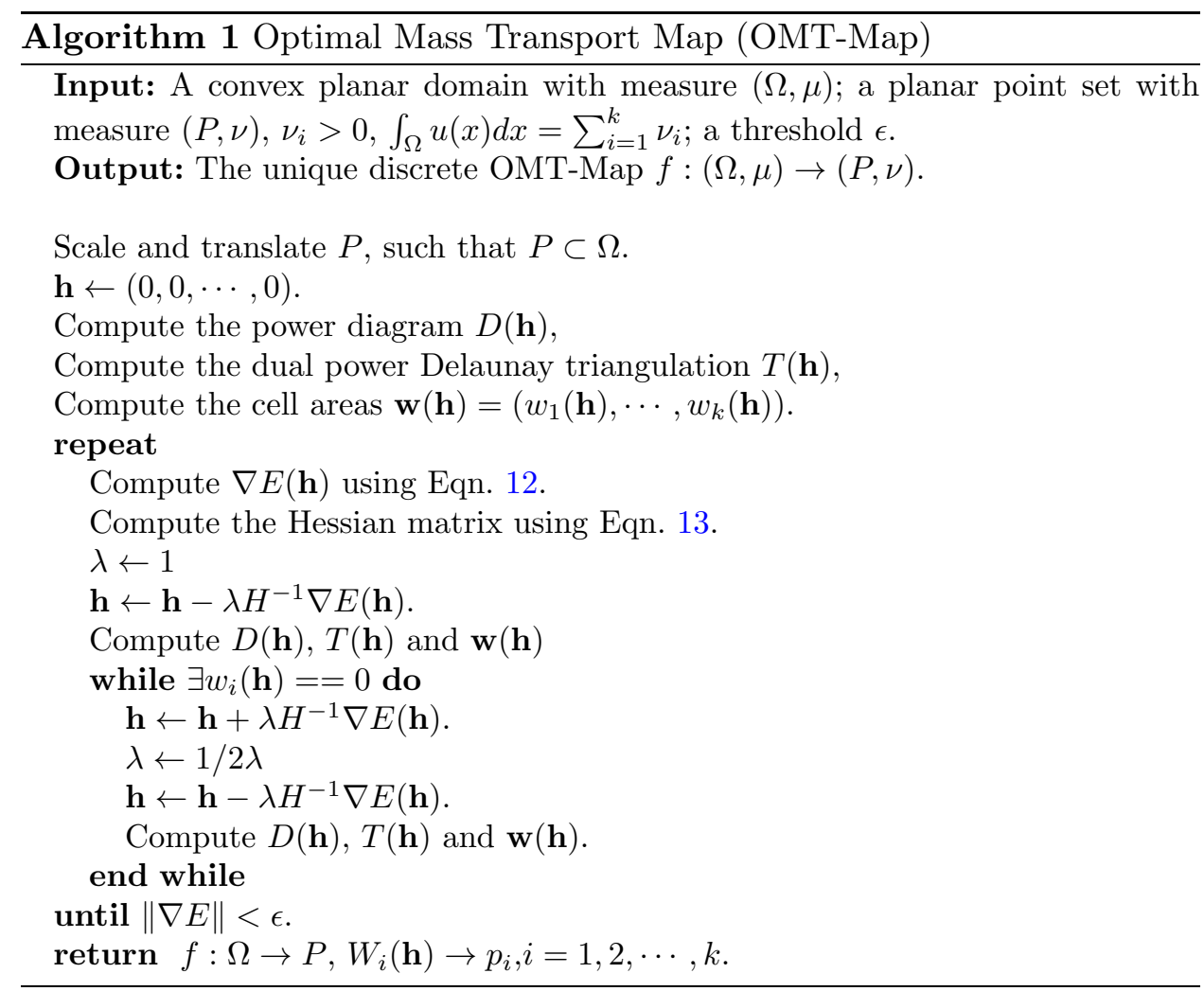


Then the Hessian matrix $H_{k}$ in Eqn. 13 is positive definite on the hyperplane $\left\{\mathbf{h} \mid \sum_{i} h_{i}=0\right\}$. At the $k+1$-step, we set the step length parameter $\lambda$ as 1 , and update the power weight vector

$$
\mathbf{h}^{k+1}=\mathbf{h}^{k}-\lambda H_{k}^{-1} \nabla E\left(\mathbf{h}^{k}\right) .
$$

Then we compute the power diagram $D\left(P, \mathbf{h}^{k+1}\right)$. If any Voronoi cell $W_{i}\left(\mathbf{h}^{k+1}\right)$ disappears, then the Hessian matrix $H_{k+1}$ will be degenerated. In this case, we shrink the step length parameter $\lambda$ to be half, $\lambda \leftarrow \frac{1}{2} \lambda$. Then we recompute $\mathbf{h}^{k+1}$ using the formula in Eqn. 14 and test again. We repeat this procedure, until all Voronoi cells in $D\left(P, \mathbf{h}^{k+1}\right)$ are non-empty. Algorithm 1 gives the implementation details.

\subsection{Conformal Wasserstein distance}

The OMT-Map algorithm can also be generalized to compute the Wasserstein distance between two surfaces. Given two topological disk surfaces $\left(M_{1}, g_{1}, p_{1}, q_{1}\right) \in \mathcal{S},\left(M_{2}, g_{2}, p_{2}, q_{2}\right) \in \mathcal{S}$ with total area $\pi$, where $\mathcal{S}$ is the normalized marked metric space. $p_{1}$ and $p_{2}$ are correspondent interior markers, and $q_{1}$ and $q_{2}$ are correspondent boundary markers. We first compute the conformal maps $\phi_{1}: M_{1} \rightarrow \mathbb{D}_{1}$ and $\phi_{2}: M_{2} \rightarrow \mathbb{D}_{2}$, where $\mathbb{D}_{1}$ and $\mathbb{D}_{2}$ are the unit planar disks with Euclidean metric $d x^{2}+d y^{2}$, such that $\phi\left(p_{1}\right)=$ $\phi\left(p_{2}\right)=(0,0)$ and $\phi\left(q_{1}\right)=\phi\left(q_{2}\right)=(1,0)$. Then we construct a convex planar domain $(\Omega, \mu)$ from $\mathbb{D}_{1}$, where $\mu$ is computed by $\mu_{(s, \mathbf{g})}:=e^{2 \lambda}(x, y) d x \wedge d y$.

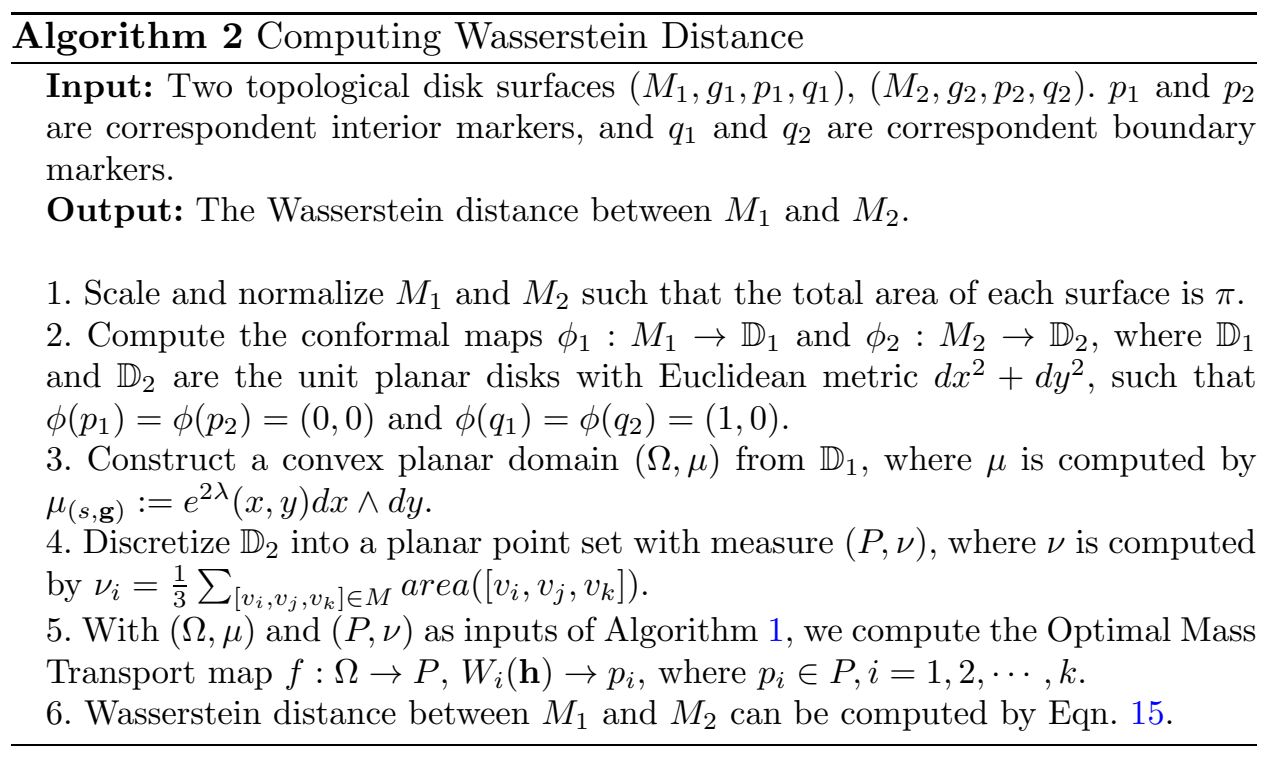


And then we discretize $\mathbb{D}_{2}$ into a planar point set with measure $(P, \nu)$, where $\nu$ is computed by $\nu_{i}=\frac{1}{3} \sum_{\left[v_{i}, v_{j}, v_{k}\right] \in M} \operatorname{area}\left(\left[v_{i}, v_{j}, v_{k}\right]\right)$. Using $(\Omega, \mu)$ and $(P, \nu)$ as inputs of Algorithm 1, we compute the Optimal Mass Transport map $f: \Omega \rightarrow P, W_{i}(\mathbf{h}) \rightarrow p_{i}$, where $p_{i} \in P, i=1,2, \cdots, k$. Therefore, the Wasserstein distance between $M_{1}$ and $M_{2}$ can be computed by

$$
\operatorname{Wasserstein}(\mu, \nu)=\sum_{i=1}^{k} \int_{W_{i}}\left(x-p_{i}\right)^{2} \mu(x) d x
$$

Algorithm 2 gives the implementation details.

\section{Experiments}

We implemented our algorithms using $\mathrm{C}++$ on Windows platform, and all the experiments were conducted on a laptop computer of Intel Core i7 CPU, $2.60 \mathrm{GHz}$ with $8 \mathrm{~GB}$ memory. In order to evaluate the performance of our proposed method, we compared our method with the method based on surface area distortion, and the method based on the curvature difference. The classification was performed between epilepsy data group and normal control group in a dataset of 40 hippocampuses, as shown in Table 1, where the geometric properties of surfaces (e.g., number of vertices, edges, and faces) were reported. The brain cortical surfaces (shown in Figure 2) and hippocampus surfaces were reconstructed from MRI images by FreeSurfer [12]. The support vector machine (SVM) [9] was employed as the classifier in the experiments, where the radial basis function (RBF) kernel was selected as

Table 1: Geometric properties of hippocampus surface

\begin{tabular}{lccc}
\hline Surface & Num. of Vertices & Num. of Edges & Num. of Faces \\
\hline Control & $10 \mathrm{~K}$ & $30 \mathrm{~K}$ & $20 \mathrm{~K}$ \\
Epilepsy & $10 \mathrm{~K}$ & $30 \mathrm{~K}$ & $20 \mathrm{~K}$ \\
\hline & & &
\end{tabular}

Figure 2: Lateral and medial views of brain cortical surfaces. 


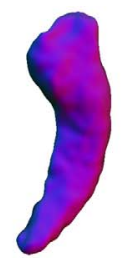

(a)

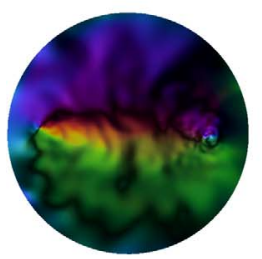

(e)

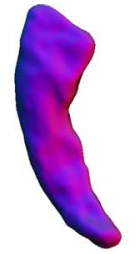

(b)

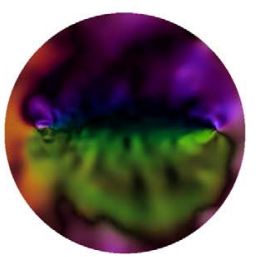

(f)

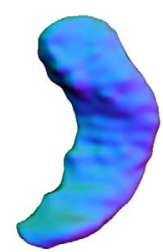

(c)

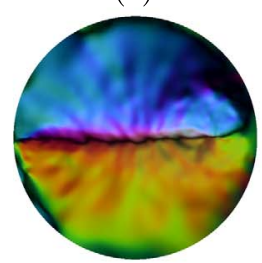

(g)

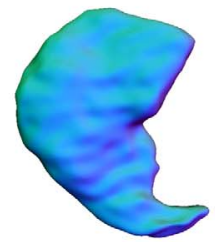

(d)

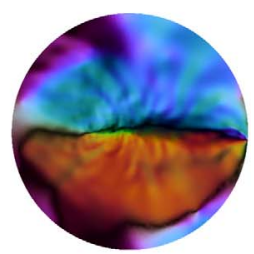

(h)

Figure 3: Hippocampal surfaces and conformal mapping results. (a)-(b) left and right hippocampus surfaces in normal control data group; (c)-(d) left and right hippocampus surfaces in epilepsy data group; (e)-(h) corresponding conformal mapping results.

the kernel function. We used $C$-SVM and selected $C=5$ for cross validation. To report the average classification performance, we randomly selected the training set each time and computed the average area under the curve (AUC) of receiver operating characteristic (ROC) over 100 times.

One important step in our proposed method is to compute a conformal mapping which maps the hippocampus surface onto the planar disk. We first sliced the hippocampus surface along a geodesic curve between two end points on the hippocampus surface, and then employed the holomorphic one-form method [16] for conformal mapping of hippocampus surface onto planar disk. Figure 3(a) and (b) show a representative pair of left and right hippocampus surfaces in normal control group, and their conformal mapping results are given in Figure 3(e) and (f), respectively. Figure 3(c) and (d) illustrate a representative pair of left and right hippocampus surfaces in epilepsy data group, with their conformal mapping results shown in Figure 3(g) and (h). To illustrate the angle preserving of the conformal mapping results, we show the conformal texture mapping result using a checkerboard and circle textures in Figure 4(a)-(d) and Figure 4(e)-(h), respectively. The texturecoordinates are defined as the plane coordinates. One texture is a regular checkerboard image and the other is an image with a collection of circles. As observed in Figure 4, the right angles at each corner of checkerboard and the circle shape are preserved. 


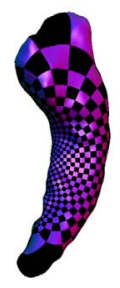

(a)

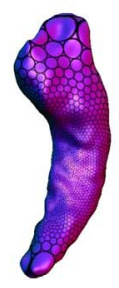

(e)

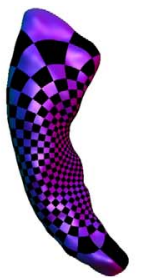

(b)

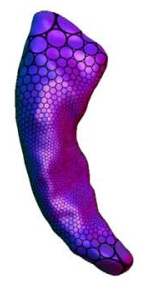

(f)

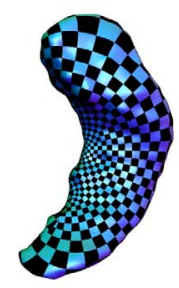

(c)

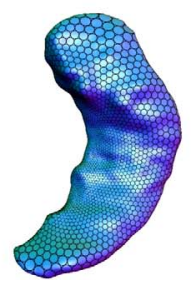

(g)

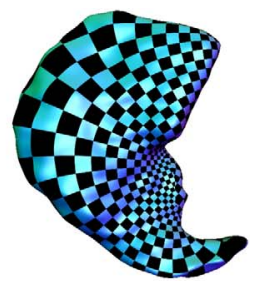

(d)

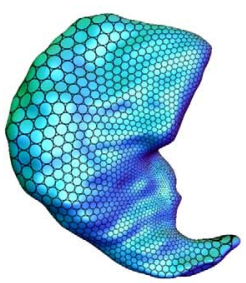

(h)

Figure 4: Conformal texture-mapping results. (a)-(d) conformal checkerboard-texture mapping results of left and right hippocampus surfaces in normal control and epilepsy data groups, respectively; (e)-(h) conformal circle-texture mapping results of left and right hippocampus surfaces in normal control and epilepsy data groups, respectively.

Previous work [8] indicates that the hippocampus is often the focus of epileptic seizures. Epilepsy can cause some deformation on hippocampus of the brain, which hence may result in shape dissimilarity between left and right hippocampus. We hypothesize that our OMT-Map based Wasserstein distance method, which intrinsically measures the dissimilarities between shapes, may help early epilepsy detection. In our following experiments, we computed the area distortion, curvature difference between registered left and right hippocampal surfaces for each data in normal control data group and in epilepsy data group. We then computed Wasserstein distance used in our proposed method as above and demonstrated the classification performance of our proposed method, in comparison to the other two methods.

Area Distortion In order to compute the area distortion between left and right hippocampal surfaces, a Jacobian determinant $J\left(v_{2}\right)$ was computed for each vertex $v_{2}$ on the right hippocampal surface, where the corresponding vertex on the left hippocampal surface is denoted as $v_{1}$. Then the local area distortion at $v_{2}$ can be computed by $\max \left(J\left(v_{2}\right), J^{-1}\left(v_{2}\right)\right)$, where $J$ is approximated by the ratio between the measure $\mu\left(v_{2}\right)$ and $\mu\left(v_{1}\right)$, and 


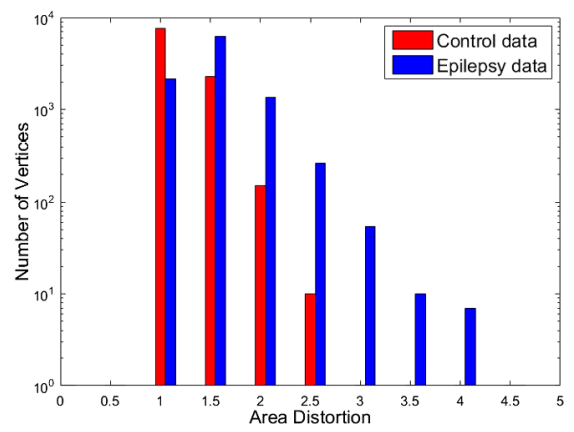

Figure 5: Histograms of area distortion for comparison between control and epilepsy data.

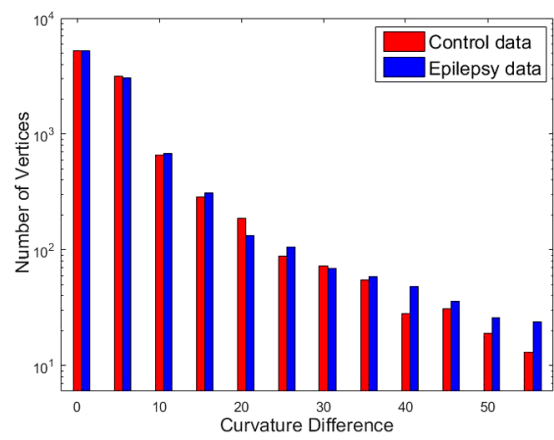

Figure 6: Histograms of curvature for comparison between control and epilepsy data.

$\mu\left(v_{i}\right)=\frac{1}{3} \sum_{j k} \operatorname{Area}\left(\left[v_{i}, v_{j}, v_{k}\right]\right)$. In Figure 5, the histogram of area distortion shows that the pair of left and right hippocampal surfaces of the normal control data produces less area distortions in comparison to the ones of the epilepsy data.

Curvature Difference A convexity measure capturing the approximation of mean curvature was employed to compute the curvature map. We computed the difference of curvature maps between the registered left and right hippocampal surfaces. Given the curvature $c 1$ of a vertex on the right hippocampal surface and the curvature $c 2$ of a corresponding vertex on the left hippocampal surface, the curvature difference is denoted as $\max \left(\frac{|c 1|}{|c 2|}, \frac{|c 2|}{|c 1|}\right)$. As shown in Figure 6, the histogram of curvature difference demonstrates that the pair of left and right hippocampal surfaces of the epilepsy data 


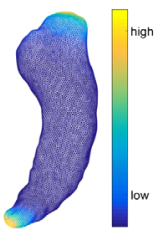

(a)

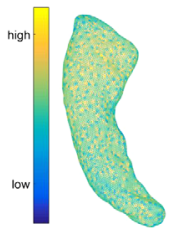

(b)

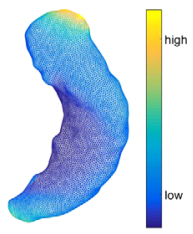

(c)

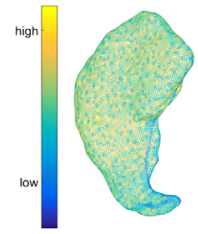

(d)

Figure 7: Measures in computation of Wasserstein distance. (a)-(b) measures on left and right hippocampus surfaces in normal control group; (c)-(d) measures on left and right hippocampus surfaces in epilepsy group.

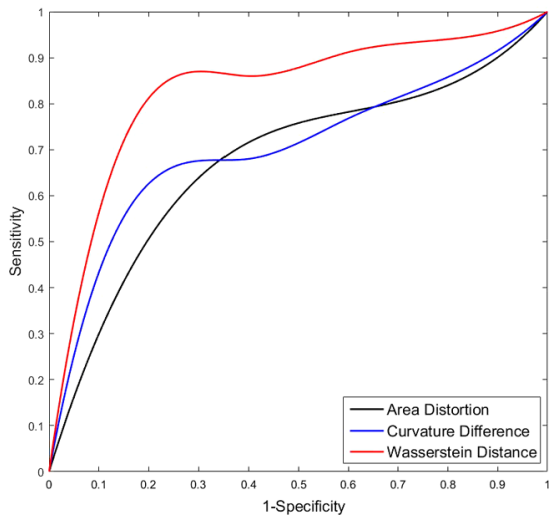

Figure 8: Comparison of average ROC curves for three methods.

yields larger curvature difference, compared with the hippocampal surfaces of the normal control data.

Wasserstein Distance Wasserstein distance is computed by measuring the minimum transportation cost induced by the optimal mass transport map between two surfaces, given two measures defined on two surfaces. Figure $7(\mathrm{a})$ and (b) show two measures on the left and the right hippocampal surfaces of a normal control data. Figure $7(\mathrm{c})$ and (d) illustrate two measures on the left and the right hippocampal surfaces of a epilepsy data. Using Algorithm 2, we can compute Wasserstein distance between two hippocampus surfaces. To demonstrate the classification performance of our proposed method, we employed SVM classifier to perform classification on a dataset including normal control data and epilepsy data. Figure 8 show the classification results by ROC curves of three different methods. As observed in 


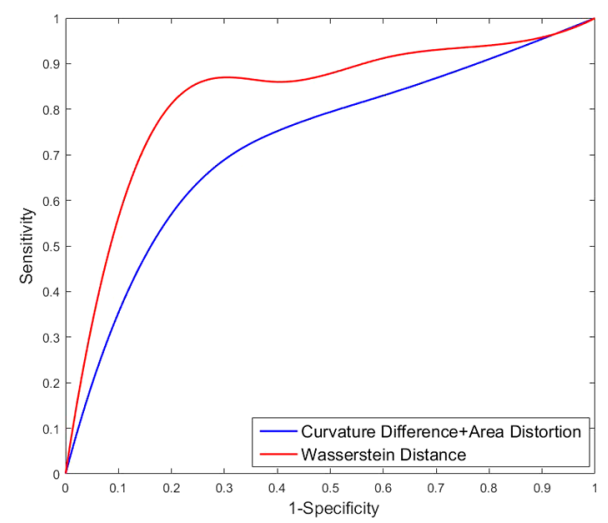

Figure 9: Comparison of average ROC curves between our method and the combination of the other two methods.

Table 2: Average AUC value

\begin{tabular}{lc}
\hline Methods & Average AUC value \\
\hline Area Distortion & 0.6948 \\
Curvature Difference & 0.7342 \\
Curvature Difference + Area Distortion & 0.7542 \\
Wasserstein Distance & 0.8834 \\
\hline
\end{tabular}

Figure 8, our OMT-Map based Wasserstein distance method outperformed the method based on surface area distortion, and the method based on the curvature difference. To quantitatively measure the classification performance, we computed the average AUC value corresponding to the ROC curves in Figure 8. Table 2 shows that our proposed method achieved 0.8838 average AUC value, which demonstrated better classification performance, in comparison with 0.6948 and 0.7342 average AUC values produced by the other two methods. Furthermore, we compared our proposed method with the combined curvature difference and area distortion based method. Figure 9 demonstrates our proposed method still achieved better classification performance, which was also evidenced by the less AUC value 0.7542 obtained by the curvature difference and area distortion based method.

\section{Conclusion}

In this work, a novel shape classification method for brain's hippocampus is proposed using Wasserstein distance based on optimal mass transport theory. The algorithms for computation of optimal mass transport map, 
OMT-Map based area preserving mapping and computation of Wasserstein distance are presented with details. Experimental evaluations on our proposed OMT-Map based Wasserstein distance method demonstrated better classification performance of our method. In the future, we will generalizes our proposed method to surfaces with more general topology.

\section{References}

[1] A. D. Alexandrov. Convex Polyhedra. Springer, 2005. MR2127379

[2] F. Aurenhammer. Power diagrams: Properties, algorithms and applications. SIAM J. Comput., 16:78-96, 1987. MR0873251

[3] Vincent Barra and Silvia Biasotti. 3d shape retrieval and classification using multiple kernel learning on extended reeb graphs. The Visual Computer, 30(11):1247-1259, 2014.

[4] Zafer Barutcuoglu and Christopher DeCoro. Hierarchical shape classification using bayesian aggregation. In IEEE International Conference on Shape Modeling and Applications, 2006, SMI 2006, pages 44-44. IEEE, 2006.

[5] Harry Blum and Roger N. Nagel. Shape description using weighted symmetric axis features. Pattern Recognition, 10(3):167-180, 1978.

[6] Nicolas Bonnotte. From Knothe's rearrangement to Brenier's optimal transport map. arXiv:1205.1099, pages 1-29, 2012.

[7] Yann Brenier. Polar factorization and monotone rearrangement of vector-valued functions. Communications on Pure and Applied Mathematics, 44(4):375-417, 1991. MR1100809

[8] B. S. Chang and D. H. Lowenstein. Epilepsy. The New England Journal of Medicine, 349:1257-1266, 2003.

[9] Chih-Chung Chang and Chih-Jen Lin. LIBSVM: A library for support vector machines. ACM Transactions on Intelligent Systems and Technology, 2:27:1-27:27, 2011. Software available at http://www.csie.ntu.edu.tw/ cjlin/libsvm.

[10] Fernando de Goes, Katherine Breeden, Victor Ostromoukhov, and Mathieu Desbrun. Blue noise through optimal transport. ACM Transactions on Graphics (TOG), 31(6):171, 2012.

[11] Fernando De Goes, David Cohen-Steiner, Pierre Alliez, and Mathieu Desbrun. An optimal transport approach to robust reconstruction and 
simplification of $2 \mathrm{~d}$ shapes. In Computer Graphics Forum, volume 30, pages 1593-1602. Wiley Online Library, 2011.

[12] Rahul S. Desikan, Florent Ségonne, Bruce Fischl, Brian T. Quinn, Bradford C. Dickerson, Deborah Blacker, Randy L. Buckner, Anders M. Dale, R. Paul Maguire, Bradley T. Hyman, Marilyn S. Albert, and Ronald J. Killiany. An automated labeling system for subdividing the human cerebral cortex on mri scans into gyral based regions of interest. NeuroImage, 31(3):968 - 980, 2006.

[13] Ayelet Dominitz and Allen Tannenbaum. Texture mapping via optimal mass transport. IEEE Transactions on Visualization and Computer Graphics, 16(3):419-433, 2010.

[14] Zhanheng Gao, Zeyun Yu, and Xiaoli Pang. A compact shape descriptor for triangular surface meshes. Computer-Aided Design, 53:62-69, 2014.

[15] Catriona D. Good, Ingrid Johnsrude, John Ashburner, Richard N. A. Henson, Karl J. Friston, and Richard S. J. Frackowiak. Cerebral asymmetry and the effects of sex and handedness on brain structure: A voxel-based morphometric analysis of 465 normal adult human brains. Neuroimage, 14(3):685-700, 2001.

[16] X. Gu and S.-T. Yau. Computing conformal structures of surfaces. Communications In Information and Systems, 2(2):121-146, 2002. MR1958012

[17] Xianfeng Gu, Feng Luo, Jian Sun, and S.-T. Yau. Variational principles for Minkowski type problems, discrete optimal transport, and discrete Monge-Amperé equations. arXiv:1302.5472, 2013.

[18] Xianfeng Gu and Shing-Tung Yau. Computational Conformal Geometry. International Press, 2008. MR2439718

[19] Steven Haker, Lei Zhu, Allen Tannenbaum, and Sigurd Angenent. Optimal mass transport for registration and warping. International Journal of Computer Vision, 60(3):225-240, 2004.

[20] Robert M. Haralick, Stanley R. Sternberg, and Xinhua Zhuang. Image analysis using mathematical morphology. IEEE Transactions on Pattern Analysis and Machine Intelligence, (4):532-550, 1987.

[21] Dominic Holland, James B. Brewer, Donald J. Hagler, Christine Fennema-Notestine, Anders M. Dale, Michael Weiner, Leon Thal, Ronald Petersen, Clifford R. Jack, William Jagust, et al. Subregional 
neuroanatomical change as a biomarker for alzheimer's disease. Proceedings of the National Academy of Sciences, 106(49):20954-20959, 2009.

[22] L. V. Kantorovich. On a problem of Monge. Uspekhi Mat. Nauk., 3:225226, 1948.

[23] Haibin Ling and David W. Jacobs. Shape classification using the innerdistance. IEEE Transactions on Pattern Analysis and Machine Intelligence, 29(2):286-299, 2007.

[24] M. Mahmoudi and G. Sapiro. Three-dimensional point cloud recognition via distributions of geometric distances. In IEEE Computer Society Conference on Computer Vision and Pattern Recognition Workshops, 2008, CVPRW '08, pages 1-8, June 2008.

[25] Li Shen, James Ford, Fillia Makedon, and Andrew Saykin. Hippocampal shape analysis: surface-based representation and classification. In Medical Imaging 2003, pages 253-264. International Society for Optics and Photonics, 2003.

[26] Li Shen, James Ford, Fillia Makedon, and Andrew Saykin. A surfacebased approach for classification of $3 \mathrm{~d}$ neuroanatomic structures. Intelligent Data Analysis, 8(6):519-542, 2004.

[27] Zhengyu Su, Wei Zeng, Rui Shi, Yalin Wang, Jian Sun, and Xianfeng $\mathrm{Gu}$. Area preserving brain mapping. In Proceedings of the IEEE Conference on Computer Vision and Pattern Recognition, pages 2235-2242, 2013.

[28] Cedric Villani. Topics in Optimal Transportation. American Mathematical Society, 2003. MR1964483

[29] Wei Zeng, Dimitris Samaras, and Xianfeng David Gu. Ricci flow for 3d shape analysis. IEEE Transactions on Pattern Analysis and Machine Intelligence, 32(4):662-677, 2010.

[30] Yun Zeng, Chaohui Wang, Yang Wang, Xianfeng Gu, Dimitris Samaras, and Nikos Paragios. Dense non-rigid surface registration using highorder graph matching. In 2010 IEEE Conference on Computer Vision and Pattern Recognition (CVPR), pages 382-389. IEEE, 2010.

Ming MA

Department of Computer Science

STONY BROOK UNIVERSITY

STONY BROOK, NY

USA

E-mail address: minma@cs.stonybrook.edu 
NA LEI

Dalian University of TEChNOLOGy

DALIAN, LIAONING

China

E-mail address: nalei@outlook.com

Kehua Su

State Key Laboratory of Software Engineering

WUHAN UNIVERSITY

WuHAN, Huibei

China

E-mail address: skhemailg@gmail.com

JUNWEI ZHANG

STONY Brook UnIVERSITY

STONY BROOK, NY

USA

E-mail address: junwei.zhang@stonybrook.edu

Chengfeng Wen

Department of Computer Science

Stony Brook University

STONY BROOK, NY

USA

E-mail address: chwen@cs.stonybrook.edu

Li CUI

School of Mathematical Sciences

Beijing Normal University

BEIJING

China

E-mail address: licuibnu@gmail.com

XIN FAN

Dalian University of Technology

Dalian, LiaOning

China

E-mail address: xin.fan@dlut.edu.cn

Xianfeng Gu

Department of Computer Science

STONY BROOK UNIVERSITY

STONY BROOK, NY

USA

E-mail address: gu@cs.sunysb.edu

Received 3 August 2015 